\title{
Estrategias de aprendizaje y rendimiento académico empleadas por estudiantes de liceos de Talca con alto y bajo puntaje del Sistema de Medición de la Calidad de la Enseñanza
}

\author{
Strategies of learning and academic performance used by high school \\ students of Talca with high and low score of the System of Measurement of \\ the Quality of Teaching
}

\author{
FRANCESCA MAGDALENA GÓMEZ TORRES \\ ANDREA ZENOBIA MERINO BRAVO \\ Psicólogas \\ Universidad Autónoma de Chile \\ andremerinob@gmail.com
}

\section{RESUMEN}

En el proceso educativo, las estrategias de aprendizaje han adquirido un gran protagonismo debido a que procesan, organizan, retienen y recuperan la información de acuerdo con los pensamientos, motivaciones y emociones de los estudiantes, además, permiten la inclusión de nuevos conocimientos y establecer relaciones con los ya adquiridos; estas estrategias generan un aprendizaje altamente significativo. El objetivo del presente estudio consistió en evaluar las estrategias de aprendizaje y rendimiento académico de estudiantes de cuarto año de enseñanza media de liceos municipalizados de la comuna de Talca que presentaron alto y bajo puntaje en el Sistema de Medición de la Calidad de la Enseñanza-SIMCE (MINEDUC 2006). Se realizó un estudio cuantitativo de diseño transversal y se seleccionó, de manera intencional, a estudiantes de cuarto medio de cuatro liceos municipalizados de la comuna de Talca, de acuerdo con el puntaje SIMCE alcanzado por los establecimientos escolares. A la muestra, se le aplicó el Inventario de Estrategias de Estudio y Aprendizaje (IEEA). Los resultados reflejaron que existe una relación entre las estrategias de aprendizaje y el rendimiento académico de los alumnos de liceos municipalizados con alto puntaje SIMCE de Talca. Además, se evidenció que los estudiantes 
pertenecientes a liceos con bajo puntaje SIMCE emplean estrategias de aprendizaje, pero estas no se relacionan con un buen rendimiento académico; asimismo, los estudiantes varones presentan mayor concentración y mejor manejo de la ansiedad que las mujeres. Sin embargo, se requiere profundizar en las estrategias de aprendizaje de alumnos cercanos al egreso de la educación media y en aquellas estrategias que no están presentes durante el proceso de estudio, así como en su relación con el éxito o fracaso escolar.

Palabras clave: estrategias de aprendizaje, calidad de la enseñanza, educación secundaria, rendimiento académico.

\section{ABSTRACT}

During the education process, learning strategies have gained great importance because they help process, organize, retain and retrieve information according to the students' thoughts, motivations, and emotions, also, they allow for the inclusion of new understanding and establish relationships with those already acquired; these strategies generate highly significant learning. The objective of the present study consist in evaluating the learning strategies and academic performance of 12th grade students in public schools from the Talca community that have high and low scores in the Measurement System of Teaching Quality-SIMCE (MINEDUC 2006). A quantitative cross-sectional design was performed and intentionally selected 12th grade students from four public high schools from the Talca community, according to the high schools' SIMCE scores. The Study and Learning Strategies Inventory (IEEA) was applied to the sample. The results showed that there is a relationship between the learning strategies and academic performance of students from high schools with high SIMCE scores in Talca. Additionally, evidence showed that students from high schools with low SIMCE scores used learning strategies, but did not relate to good academic performance; also male students showed higher concentration and better anxiety management then female students. However, it is necessary to intensify learning strategies for students closer to their high school graduation and intensify strategies that are not used during the learning process as well as the relationship it has with the school success or failure.

Key words: learning Strategies, teaching quality, High school education, academic performance. 


\section{INTRODUCCIÓN}

Las estrategias de aprendizaje se pueden entender como todo tipo de pensamiento, acciones, comportamientos, creencias e incluso emociones que permiten y apoyan la adquisición de información, además de favorecer la asociación con el conocimiento previo y la recuperación de la información ya existente (Pizano, 2004). A través de las estrategias, se puede procesar, organizar, retener y recuperar el material informativo que se tiene que aprender, a la vez que se planifica, regula y evalúa esos mismos procesos en función del objetivo previamente trazado 0 exigido por las demandas de la tarea (Woolfolk, 2015).

Las definiciones expuestas ponen de relieve dos notas importantes al momento de establecer el concepto de estrategias. En primer lugar, se trata de actividades u operaciones mentales que realiza el estudiante para mejorar el aprendizaje y, en segundo lugar, las estrategias tienen un carácter intencional o propositivo, e implican, por tanto, un plan de acción (Pizano, 2004). Otros autores concuerdan con la idea de que las estrategias de aprendizaje son acciones que parten de la iniciativa del alumno, están constituidas por una secuencia de actividades, se encuentran controladas por el sujeto que aprende, y son, generalmente, deliberadas y planificadas por el propio estudiante.

Por último, la psicología evolutiva y la psicología cognitiva concuerdan en que las estrategias de aprendizaje son un conjunto de habilidades y procedimientos de tipo general; sumado a esto, una corriente más reciente como lo es la psicología de la educación considera la influencia de lo social-contextual sobre las estructuras cognitivas del aprendiz (Monereo, Pozo y Castelló, 2004).

Dentro de las razones explicativas del surgimiento de este concepto se pueden mencionar: la falta de entrenamiento de las habilidades de aprendizaje de los estudiantes de enseñanza básica y media, la comprobación de diferentes estrategias entre alumnos con éxito y alumnos con bajo rendimiento, el descenso de la población estudiantil y la consiguiente flexibilización de los sistemas de acceso a los estudios superiores; sumado a esto, también se destaca el apoyo científico, el cual ha hecho su aporte con base a los estudios sobre la inteligencia, el nuevo concepto de aprendizaje y las experiencias educativas en ambientes naturales. 
En el uso de las estrategias de aprendizaje cobra importancia el manejo y control de las conductas ansiosas, puesto que se orienta hacia la percepción de los eventos estresantes y la reacción que se tiene ante ellos.

La importancia de los componentes afectivos-motivacionales determinan, en gran medida, las estrategias específicas que los estudiantes utilizan en tareas de aprendizajes particulares. Por eso, se entiende que la motivación es un componente necesario de la conducta estratégica y un requisito previo para utilizar estrategias. Esto, indica que los alumnos suelen disponer de recursos para mejorar el aprendizaje, aunque la puesta en marcha de los mismos depende, entre otros factores, de las metas que persigue, independientemente de que estas sean de tipo académicas o sean propósitos e intenciones que guíen su conducta ante una tarea de aprendizaje en particular.

El interés de la enseñanza de estrategias reside en la potencialidad que se da al alumno para controlar y regular sus procesos mentales de aprendizaje, lo que habría de permitirle mejorar su rendimiento y, sobre todo, su capacidad de aprender autónomamente a partir de sus propios recursos. A partir de esto, se logra la unión de dos enfoques explicativos del proceso de estudio: enfoque asociacionista y cognoscitivo, los cuales plantean el hecho de que el estudiante debe utilizar en conjunto procesos internos y externos para adquirir conocimiento y desarrollar habilidades y destrezas, además de incorporar hábitos de organización y planificación de su estudio y el tiempo dedicado a ello (Andrade, 2001).

Muñoz (2005) en su estudio describe las estrategias de aprendizaje de 45 estudiantes universitarias de primer año de educación parvularia de la Universidad Autónoma del Sur de Talca. Para la recolección de datos se utilizó la versión mexicana del Inventario de Estrategias de Estudio y Aprendizaje (IEEA, versión castellana del LASSI). Los resultados arrojaron que las alumnas presentaron un puntaje bajo la media en lo que se refiere a: las categorías de motivación, estrategias para la preparación y presentación de exámenes y adecuación a la ansiedad, correlacionando significativamente la categoría de actitud e interés con el rendimiento en los exámenes.

Del mismo modo, Muñoz y Lucero (2006) realizaron una investigación con una muestra constituida por 100 estudiantes que tomaron la asignatura de procesos afectivos. Los instrumentos utilizados fueron la Escala General de Goldberg (versión abreviada GHQ-12) y el Inventario de Estrategias de Estudio y Aprendizaje (IEEA, versión castellana del LASSI). Estos instrumentos se aplicaron al inicio y 
al final del semestre, con la finalidad de conocer las estrategias de aprendizaje y salud mental de los alumnos durante su proceso de formación, en el marco de dicha asignatura. Los resultados obtenidos reflejaron que los hombres utilizan menos estrategias de estudio que las mujeres, quienes presentan una elevada ansiedad. En relación a la escala de ansiedad y concentración, se observó una diferencia significativa entre la primera y segunda aplicación, ya que en esta última la ansiedad aumentó y la concentración disminuyó, probablemente, debido a la tensión de de las evaluaciones finales del semestre.

Por otro lado, Zulia (2006) realizó un estudio de caso hermenéutico en un instituto universitario en Maracay, con alumnos del tercer semestre de Farmacología. Los resultados arrojaron que se utilizan estrategias cognitivas y metacognitivas que permiten desarrollar en el alumnado habilidades de aprendizaje que sistematizan su proceso intelectual.

Proctor y et al. (2006) investigaron una población de 263 alumnos pertenecientes a una importante universidad del sureste de Estados Unidos; ese corpus fue divido en cuatro grupos: de acuerdo al éxito académico (alto y bajo) y según las dificultades en el aprendizaje (diagnosticada y no diagnosticada). Los resultados se obtuvieron mediante el Learning and Study Strategies Inventory (LASSI) y arrojaron que los tres últimos grupos presentan debilidades en: ansiedad, concentración, motivación, selección de ideas principales y estrategias de evaluación. De igual manera, se llegó a la conclusión de que ninguno de los grupos presenta fortalezas con respecto al manejo de las estrategias de estudio, destacándose solo el grupo de éxito académico bajo, debido a presentar mayores ayudas de estudio.

De la misma forma, Cabrera, García, Torbay y Rodríguez (2007) en su trabajo "Estructura factorial y fiabilidad de un cuestionario de estrategias de aprendizaje en universitarios: CEA-U", se basó en el modelo NOTICE, con el objetivo de generar un cuestionario sencillo y fiable que sirva para conocer las estrategias de aprendizaje utilizadas por los estudiantes. Este se aplicó a una muestra de 1105 alumnos de segundo ciclo de carrera de la Universidad de La Laguna, España. Los resultados muestran que los alumnos cuentan con tres mecanismos diferentes para afrontar una tarea: cognitivo (centrado en la comprensión y aprendizaje del contenido), organizativo (enfocado en la tarea de estudio y organización de la información) y aproximativo (referido a los mecanismos emocionales que utiliza el estudiante para alcanzar la tarea de estudio). 
Por otro lado, el objetivo del siguiente trabajo fue evaluar las estrategias de aprendizaje y rendimiento académico de los estudiantes de cuarto año de enseñanza media de liceos municipalizados con alto y bajo puntaje SIMCE, pertenecientes a la comuna de Talca del año 2007. También, se describe la relación entre estrategias de aprendizaje y rendimiento académico con base en los resultados obtenidos en la prueba SIMCE y se comparan las estrategias de aprendizaje con rendimiento académico de alumnos pertenecientes a liceos con alto y bajo puntaje SIMCE, además de indagar sobre la variable del género. El estudio se realizó a través de una metodología cuantitativa, con un diseño de tipo exploratorio-descriptivo-correlacional. La muestra estuvo conformada por 397 alumnos pertenecientes a cuatro liceos municipalizados de la comuna de Talca, de ellos 236 son estudiantes de liceos con alto puntaje SIMCE y 161 alumnos de liceos con bajo puntaje SIMCE.

En síntesis, se puede observar que los estudiantes no utilizan la totalidad de las estrategias de aprendizaje, sin embargo, existen estrategias que se encuentran más presentes al momento de estudiar, las cuales conllevan a que los estudiantes logren alcanzar un rendimiento académico superior en contraste con quienes presentan un poco o escaso uso de dichas estrategias. De la misma forma, dentro de las investigaciones citadas anteriormente, se observó concordancia en los resultados obtenidos por la escala de ansiedad, ya que, en general, se presentó un débil manejo de ella por parte de los estudiantes. También, se evidenció una gran influencia entre las distintas estrategias de aprendizaje, pues si una de ellas no se utiliza con frecuencia se puede afectar el uso frecuente de las otras estrategias, y por consiguiente intervenir en el proceso de aprendizaje realizado por el estudiante.

\section{MÉTODO}

La investigación consistió en un estudio cuantitativo, de diseño transversal, exploratorio, descriptivo y correlacional, debido a que no existen antecedentes previos en relación a la temática indagada, por lo que adquiere un carácter novedoso, pues no se ha estudiado si existe relación entre las estrategias de aprendizaje y el rendimiento académico en estudiantes según puntaje alcanzado en la prueba SIMCE en sus colegios.

La aplicación del instrumento utilizado se realizó en los meses de junio, julio y agosto del año 2007 en cuatro liceos municipalizados pertenecientes a la comuna 
de Talca, los cuales fueron seleccionados de acuerdo con el puntaje obtenido en la prueba SIMCE del año 2005. Esto con la finalidad de conocer las estrategias de aprendizaje utilizadas por los alumnos y su relación con el rendimiento académico, considerando este como el promedio con el cual ingresaron a cuarto medio, los cuales fluctúan entre 3,7 a 6,7, concentrándose la mayoría de la muestra seleccionado entre los promedios 5,0 y 5,3 .

Las variables a considerar corresponden a las diez escalas evaluadas por el Inventario de Estrategias de Estudio y Aprendizaje (IEEA), liceos con SIMCE de alto y bajo puntaje, tipo de enseñanza del liceo (científico humanista o técnico profesional), sexo y rendimiento académico.

La población corresponde a 27.447 alumnos aproximadamente adscritos a los liceos municipalizados de la comuna de Talca. De acuerdo al puntaje obtenido en la prueba SIMCE en el año 2005, 5.238 pertenecen a los liceos seleccionados: dos de bajo puntaje SIMCE (cuyo promedio entre ambos es de 210) y dos de alto puntaje (con un promedio de 274). El Liceo 1, con 195 puntos de promedio en la prueba SIMCE posee una población de 652 alumnos, de los cuales 82 son estudiantes de cuarto medio; el liceo 2, con 225 puntos de promedio en la prueba SIMCE, tiene una población de 862 alumnos, de ellos 142 cursan cuarto medio; el liceo 3, con 283 puntos de promedio en la prueba SIMCE, posee una población de 1575 alumnos, de los cuales 495 corresponden a estudiantes de cuarto medio; y el liceo 4, con 265 puntos en la prueba SIMCE, que cuenta con una población de 2149 alumnos, de ellos 477 cursan cuarto medio.

La muestra fue seleccionada de modo intencional y de acuerdo con la asistencia del día de la aplicación correspondiente a cada curso, quedando constituida por 397 alumnos, cuyas edades fluctúan entre 17 y 20 años. De esa muestra, 213 personas eran mujeres y 184 hombres; además, 236 eran jóvenes de los liceos con alto puntaje SIMCE y 161 jóvenes a los liceos con bajo puntaje SIMCE.

Antes de la aplicación del instrumento IEEA, se entregó una carta de consentimiento a cada director de los establecimientos seleccionados, previa conversación con cada uno de ellos. En primera instancia, se aplicó el instrumento en el liceo 1 entre los días 08 y 12 de junio a los tres cuartos medios existentes. Luego, el instrumento IEEA fue implementado en el liceo 2 el día 22 de junio a los seis cuartos medios existentes, por lo que se requirió la colaboración de una persona externa, pues solo se contaba con la jornada de la mañana de ese día. En relación con el liceo 3, el instrumento IEEA se aplicó el 11 de julio, pero solo a 
tres de los cuartos medios, debido a que el establecimiento se encontraba durante el periodo de evaluaciones y esas secciones eran las que contaban con el tiempo para ser participes del presente estudio. Finalmente, se aplicó el instrumento IEEA al liceo 4, los días 06, 07 y 08 de agosto en un curso de cada especialidad, estos fueron elegidos aleatoriamente.

La aplicación del IEEA comenzó con una descripción del inventario. Luego, a cada uno de los alumnos presentes se les entregó el instrumento y se señaló que deberían tardar entre 20 y 30 minutos en responderlo. En cuanto a la actitud de los estudiantes ante la aplicación del inventario, esta fue de interés y cooperación, incluso realizaron preguntas para aclarar las dudas con respecto a los ítem evaluados. Una vez que los alumnos terminaron de responder el inventario, se dieron los agradecimientos, tanto a los alumnos por su colaboración, como al profesor presente por su disponibilidad.

Cabe destacar que, Badenier (2002) realizó una investigación para validar la confiabilidad y validez del Learning and Study Strategies Inventory (LASSI) creado por Weinstein, Palmer \& Schulte en 1987. Para ello, utilizó la metodología cualitativa, con una muestra de 565 alumnos de cuarto año de enseñanza media, elegida de modo intencional. A esos individuos se les aplicó el inventario y luego se estimó la confiabilidad y validez del instrumento, obteniendo como resultado una confiabilidad semejante a la versión original $(0,85)$, y a las versiones posteriores en castellano, validando así cuatro escalas: estrategias cognitivas, estrategias de ansiedad, estrategias motivacionales y estrategias de hábitos.

El IEEA consta de un cuestionario de 77 preguntas, creado por Weinstein, Schulte \& Valenzuela (1995). Para ser respondido, se utiliza una escala tipo Likert de cinco puntos, que mide diferentes variables que están vinculadas a las estrategias de aprendizaje y de estudio, a través de las siguientes escalas:

- Actitud (ACT): actitudes e interés.

- Motivación (MOT): motivación, diligencia, autodisciplina y voluntad para trabajar con empeño.

- Administración del tiempo (ATI): administración del tiempo en la realización de actividades académicas.

- Ansiedad (ANS): ansiedad y preocupación por el trabajo escolar. 
- Concentración (CON): concentración y atención en las actividades académicas.

- Procesamiento de la información (PIN): procesamiento de la información, adquisición de conocimiento y razonamiento.

- Selección de ideas principales (SIP): selección de ideas principales y reconocimiento de información importante.

- Ayuda de estudio (AES): ayudas (técnica y materiales) de estudio.

- Autoevaluación (AEV): autoevaluación, repaso y preparación de clases.

- Preparación y presentación de exámenes (PPE): estrategias para la preparación y presentación de exámenes.

El análisis de los datos obtenidos se realizó a través de estadística descriptiva e inferencial. Al observar una distribución normal, se aplicaron correlaciones $r$ de Pearson y comparación de medias con prueba $T$, utilizando los programas computacional de Excel y Statistical Product and Service Solutions (SPSS), versión 11.5 .

\section{RESULTADOS}

En la tabla 1 se aprecia el puntaje promedio obtenido en el IEEA por cada uno de los liceos seleccionados ( 1 y 2 = bajo SIMCE técnico profesional y científico humanista; 3 = alto SIMCE científico humanista; 4 = Alto SIMCE técnico profesional). Además, se puede visualizar que los liceos 1, 2 y 4 presentan puntajes sobre la media en las escalas de administración del tiempo y concentración, destacándose el liceo 1 en la escala de actitud e interés, debido a que posee un puntaje sobre la media esperada en esa escala.

En relación a las escalas de procesamiento de la información, selección de ideas principales, ayudas de estudio, autoevaluación, estrategias para la preparación y presentación de exámenes y motivación, todos los liceos tuvieron puntajes bajo la media esperada. De igual manera, los alumnos pertenecientes a los cuatro liceos presentan puntajes bajo la media esperada en la escala de ansiedad, lo que indica que estos mantienen un débil control de esta. 
Tabla 1

Resultados descriptivos por colegios respecto de medias.

\begin{tabular}{|c|c|c|c|c|c|c|c|}
\hline & Liceos* $^{*}$ & $\mathrm{~N}$ & Media & Desviación típ. & $\begin{array}{l}\text { Error típ. de la } \\
\text { media }\end{array}$ & $\begin{array}{c}\% \text { alumnos } \\
\text { bajo media } \\
\text { esperada }\end{array}$ & $\begin{array}{c}\text { Media } \\
\text { esperada en } \\
\text { el test }\end{array}$ \\
\hline \multirow{4}{*}{ ACT } & 1 & 51 & 32,94 & 5,522 & ,773 & $35,3 \%$ & \multirow{4}{*}{32} \\
\hline & 2 & 110 & 27,22 & 4,602 & ,439 & $83,6 \%$ & \\
\hline & 3 & 105 & 24,89 & 5,373 & ,524 & $95,2 \%$ & \\
\hline & 4 & 131 & 28,09 & 4,618 & ,404 & $72,5 \%$ & \\
\hline \multirow{4}{*}{ MOT } & 1 & 51 & 25,43 & 5,108 & ,715 & $82,4 \%$ & \multirow{4}{*}{31} \\
\hline & 2 & 110 & 26,42 & 5,199 & ,496 & $80,9 \%$ & \\
\hline & 3 & 105 & 25,96 & 4,536 & ,443 & $84,8 \%$ & \\
\hline & 4 & 131 & 26,71 & 4,825 & ,422 & $77,9 \%$ & \\
\hline \multirow{4}{*}{ ATI } & 1 & 51 & 25,20 & 7,321 & 1,025 & $33,3 \%$ & \multirow{4}{*}{23} \\
\hline & 2 & 110 & 23,99 & 4,889 & ,466 & $34,5 \%$ & \\
\hline & 3 & 105 & 21,46 & 4,381 & ,428 & $63,8 \%$ & \\
\hline & 4 & 131 & 23,82 & 4,740 & ,414 & $32,8 \%$ & \\
\hline \multirow{4}{*}{ ANS } & 1 & 51 & 24,24 & 4,274 & ,598 & $64,7 \%$ & \multirow{4}{*}{26} \\
\hline & 2 & 110 & 23,29 & 5,261 &, 502 & $67,3 \%$ & \\
\hline & 3 & 105 & 22,11 & 5,537 &, 540 & $67,5 \%$ & \\
\hline & 4 & 131 & 22,81 & 5,006 & ,437 & $71,0 \%$ & \\
\hline \multirow{4}{*}{ CON } & 1 & 51 & 28,39 & 10,072 & 1,410 & $41,2 \%$ & \multirow{4}{*}{25} \\
\hline & 2 & 110 & 29,33 & 10,106 & ,964 & $38,2 \%$ & \\
\hline & 3 & 105 & 23,68 & 5,582 & ,545 & $47,6 \%$ & \\
\hline & 4 & 131 & 25,95 & 7,269 & ,635 & $41,2 \%$ & \\
\hline \multirow{4}{*}{ PIN } & 1 & 51 & 25,20 & 5,492 & ,769 & $64,7 \%$ & \multirow{4}{*}{27} \\
\hline & 2 & 110 & 26,56 & 5,508 & ,525 & $56,4 \%$ & \\
\hline & 3 & 105 & 26,83 & 4,763 & ,465 & $51,4 \%$ & \\
\hline & 4 & 131 & 26,66 & 5,227 & ,457 & $47,3 \%$ & \\
\hline \multirow{4}{*}{ SIP } & 1 & 51 & 17,39 & 2,721 & 381 & $43,1 \%$ & \multirow{4}{*}{18} \\
\hline & 2 & 110 & 17,75 & 3,584 & ,342 & $41,1 \%$ & \\
\hline & 3 & 105 & 17,20 & 3,332 & ,325 & $55,2 \%$ & \\
\hline & 4 & 131 & 17,50 & 2,799 & 245 & $48,9 \%$ & \\
\hline \multirow{4}{*}{ AES } & 1 & 51 & 21,20 & 5,115 & ,716 & $76,5 \%$ & \multirow{4}{*}{25} \\
\hline & 2 & 110 & 21,50 & 4,804 & ,458 & $75,5 \%$ & \\
\hline & 3 & 105 & 21,77 & 5,762 & ,562 & $68,6 \%$ & \\
\hline & 4 & 131 & 22,25 & 5,089 & ,445 & $71,0 \%$ & \\
\hline
\end{tabular}




\begin{tabular}{|c|c|c|c|c|c|c|c|}
\hline & Liceos* $^{*}$ & $\mathrm{~N}$ & Media & Desviación típ. & $\begin{array}{l}\text { Error típ. de la } \\
\text { media }\end{array}$ & $\begin{array}{c}\text { \% alumnos } \\
\text { bajo media } \\
\text { esperada }\end{array}$ & $\begin{array}{c}\text { Media } \\
\text { esperada en } \\
\text { el test }\end{array}$ \\
\hline \multirow{4}{*}{ AEV } & 1 & 51 & 22,96 & 4,968 & ,696 & $64,7 \%$ & \multirow{4}{*}{25} \\
\hline & 2 & 110 & 23,93 & 4,645 & ,443 & $53,6 \%$ & \\
\hline & 3 & 105 & 22,52 & 4,324 & ,422 & $69,5 \%$ & \\
\hline & 4 & 131 & 23,44 & 5,350 & ,467 & $58,8 \%$ & \\
\hline \multirow{4}{*}{ PPE } & 1 & 51 & 27,49 & 4,027 &, 564 & $72,5 \%$ & \multirow{4}{*}{30} \\
\hline & 2 & 110 & 27,84 & 5,354 &, 511 & $57,3 \%$ & \\
\hline & 3 & 105 & 28,00 & 5,097 & ,497 & $60,0 \%$ & \\
\hline & 4 & 131 & 28,43 & 4,474 & ,391 & $56,5 \%$ & \\
\hline
\end{tabular}

1 y 2 = bajo SIMCE técnico profesional y científico humanista; 3 = alto SIMCE científico humanista; 4 = Alto SIMCE técnico profesional

En la Tabla 2, se puede apreciar el puntaje promedio obtenido en el IEEA por puntaje SIMCE. Además se observa que los liceos con rendimiento bajo (2) presentan puntajes sobre la media esperada en las escalas de administración del tiempo y concentración. Asimismo, los puntajes alto y bajo SIMCE (1 = alto SIMCE y 2 = bajo SIMCE) poseen un rendimiento bajo la media esperada en actitud e interés, motivación, procesamiento de la información, selección de ideas principales, ayudas de estudio, autoevaluación, estrategias para la preparación y presentación de exámenes y ansiedad, indicando esta última que presentan un débil manejo. 
Tabla 2

Resultados descriptivos por puntaje SIMCE respecto de medias.

\begin{tabular}{|c|c|c|c|c|c|c|c|}
\hline & SIMCE* & $\mathrm{N}$ & Media & Desviación típ. & $\begin{array}{l}\text { Error típ. de la } \\
\text { media }\end{array}$ & $\begin{array}{c}\% \text { alumnos } \\
\text { bajo media } \\
\text { esperada }\end{array}$ & $\begin{array}{c}\text { Media } \\
\text { esperada en } \\
\text { el test }\end{array}$ \\
\hline \multirow{2}{*}{ ACT } & 1 & 236 & 26,67 & 5,208 & ,339 & $82,6 \%$ & \multirow{2}{*}{32} \\
\hline & 2 & 161 & 29,03 & 5,576 & ,439 & $68,3 \%$ & \\
\hline \multirow{2}{*}{ MOT } & 1 & 236 & 26,38 & 4,704 & ,306 & $80,9 \%$ & \multirow{2}{*}{31} \\
\hline & 2 & 161 & 26,11 & 5,175 & ,408 & $81,4 \%$ & \\
\hline \multirow{2}{*}{ ATI } & 1 & 236 & 22,77 & 4,724 & ,307 & $46,6 \%$ & \multirow{2}{*}{23} \\
\hline & 2 & 161 & 24,37 & 5,775 & ,455 & $34,2 \%$ & \\
\hline \multirow{2}{*}{ ANS } & 1 & 236 & 22,50 & 5,249 & ,342 & $70,3 \%$ & \multirow{2}{*}{26} \\
\hline & 2 & 161 & 23,59 & 4,976 & , 392 & $66,5 \%$ & \\
\hline \multirow{2}{*}{ CON } & 1 & 236 & 24,94 & 6,657 & ,433 & $44,1 \%$ & \multirow{2}{*}{25} \\
\hline & 2 & 161 & 29,03 & 10,073 & ,794 & $39,1 \%$ & \\
\hline \multirow{2}{*}{ PIN } & 1 & 236 & 26,73 & 5,016 & ,327 & $49,2 \%$ & \multirow{2}{*}{27} \\
\hline & 2 & 161 & 26,13 & 5,523 & ,435 & $59,0 \%$ & \\
\hline \multirow{2}{*}{ SIP } & 1 & 236 & 17,36 & 3,045 & , 198 & $51,7 \%$ & \multirow{2}{*}{18} \\
\hline & 2 & 161 & 17,64 & 3,331 & ,263 & $47,2 \%$ & \\
\hline \multirow{2}{*}{ AES } & 1 & 236 & 22,04 & 5,392 & ,351 & $69,9 \%$ & \multirow{2}{*}{25} \\
\hline & 2 & 161 & 21,40 & 4,891 & ,385 & $75,8 \%$ & \\
\hline \multirow{2}{*}{ AEV } & 1 & 236 & 23,03 & 4,931 & ,321 & $63,6 \%$ & \multirow{2}{*}{25} \\
\hline & 2 & 161 & 23,62 & 4,755 & 375 & $57,1 \%$ & \\
\hline \multirow{2}{*}{ PPE } & 1 & 236 & 28,24 & 4,756 & ,310 & $58,1 \%$ & \multirow{2}{*}{30} \\
\hline & 2 & 161 & 27,73 & 4,962 & ,391 & $62,1 \%$ & \\
\hline
\end{tabular}

1 = alto SIMCE y 2 = bajo SIMCE

En la Tabla 3, se puede apreciar el puntaje promedio obtenido en el IEEA por la combinación sexo-SIMCE bajo 0 alto. Se evidencia que tanto hombres pertenecientes a liceos con alto SIMCE y mujeres y hombres pertenecientes a liceos con bajo SIMCE tienen rendimiento sobre la media esperada en la escala de concentración; las mujeres y hombres adscritos a liceos con bajo SIMCE presentan puntajes sobre la media en la escala de administración del tiempo, al igual que los hombres de los liceos con alto SIMCE, quienes demuestran parámetros sobre la media esperada en la escala de procesamiento de la información. En general, todos los alumnos, tanto hombres como mujeres, pertenecientes a liceos con alto y bajo SIMCE poseen puntajes bajo la media esperada en la escala de actitud e interés, motivación, selección de ideas principales, ayudas de estudio, 
autoevaluación, estrategias para la preparación y presentación de exámenes y ansiedad, indicando esta última que presentan un débil control de ella.

\section{Tabla 3}

Resultados descriptivos por sexo-SIMCE respecto de medias.

\begin{tabular}{|c|c|c|c|c|c|c|c|}
\hline & Sexo/SIMCE & $\mathrm{N}$ & Media & $\begin{array}{c}\text { Desviación } \\
\text { típ. }\end{array}$ & $\begin{array}{l}\text { Error típ. de } \\
\text { la media }\end{array}$ & $\begin{array}{c}\% \text { alumnos } \\
\text { bajo media } \\
\text { esperada }\end{array}$ & $\begin{array}{c}\text { Media } \\
\text { esperada } \\
\text { en el test }\end{array}$ \\
\hline \multirow{4}{*}{ ACT } & Mujer alto SIMCE & 139 & 27,27 & 5,313 & ,451 & $81,3 \%$ & \multirow{4}{*}{32} \\
\hline & Hombre alto SIMCE & 97 & 25,80 & 4,955 &, 503 & $84,5 \%$ & \\
\hline & Mujer bajo SIMCE & 74 & 28,77 & 5,925 & ,689 & $68,9 \%$ & \\
\hline & Hombre bajo SIMCE & 87 & 29,25 & 5,285 & ,567 & $67,8 \%$ & \\
\hline \multirow{4}{*}{ MOT } & Mujer alto SIMCE & 139 & 26,90 & 4,677 & ,397 & $77,0 \%$ & \multirow{4}{*}{31} \\
\hline & Hombre alto SIMCE & 97 & 25,63 & 4,664 & ,474 & $86,6 \%$ & \\
\hline & Mujer bajo SIMCE & 74 & 26,46 & 5,187 & ,603 & $75,5 \%$ & \\
\hline & Hombre bajo SIMCE & 87 & 25,80 & 5,176 &, 555 & $86,2 \%$ & \\
\hline \multirow{4}{*}{ ATI } & Mujer alto SIMCE & 139 & 22,96 & 4,735 & ,402 & $41,0 \%$ & \multirow{4}{*}{23} \\
\hline & Hombre alto SIMCE & 97 & 22,51 & 4,719 & ,479 & $54,6 \%$ & \\
\hline & Mujer bajo SIMCE & 74 & 25,39 & 6,826 & ,794 & $27,0 \%$ & \\
\hline & Hombre bajo SIMCE & 87 & 23,51 & 4,564 & ,489 & $40,2 \%$ & \\
\hline \multirow{4}{*}{ ANS } & Mujer alto SIMCE & 139 & 21,51 & 5,042 & ,428 & $77,7 \%$ & \multirow{4}{*}{26} \\
\hline & Hombre alto SIMCE & 97 & 23,92 & 5,239 & ,532 & $59,8 \%$ & \\
\hline & Mujer bajo SIMCE & 74 & 23,18 & 5,201 & ,605 & $64,9 \%$ & \\
\hline & Hombre bajo SIMCE & 87 & 23,94 & 4,777 &, 512 & $67,8 \%$ & \\
\hline \multirow{4}{*}{ CON } & Mujer alto SIMCE & 139 & 24,64 & 7,124 & ,604 & $48,9 \%$ & \multirow{4}{*}{25} \\
\hline & Hombre alto SIMCE & 97 & 25,37 & 5,932 & ,602 & $37,1 \%$ & \\
\hline & Mujer bajo SIMCE & 74 & 26,39 & 7,844 & ,912 & $44,6 \%$ & \\
\hline & Hombre bajo SIMCE & 87 & 31,28 & 11,201 & 1,201 & $34,5 \%$ & \\
\hline \multirow{4}{*}{ PIN } & Mujer alto SIMCE & 139 & 26,17 & 5,026 & ,426 & $51,1 \%$ & \multirow{4}{*}{27} \\
\hline & Hombre alto SIMCE & 97 & 27,54 & 4,916 & ,499 & $46,4 \%$ & \\
\hline & Mujer bajo SIMCE & 74 & 26,61 & 6,018 & ,700 & $50,0 \%$ & \\
\hline & Hombre bajo SIMCE & 87 & 25,72 & 5,064 &, 543 & $66,7 \%$ & \\
\hline \multirow{4}{*}{ SIP } & Mujer alto SIMCE & 139 & 17,24 & 2,891 & ,245 & $53,2 \%$ & \multirow{4}{*}{18} \\
\hline & Hombre alto SIMCE & 97 & 17,54 & 3,260 & ,331 & $49,5 \%$ & \\
\hline & Mujer bajo SIMCE & 74 & 17,92 & 3,226 & ,375 & $45,9 \%$ & \\
\hline & Hombre bajo SIMCE & 87 & 17,40 & 3,418 & ,366 & $48,3 \%$ & \\
\hline
\end{tabular}




\begin{tabular}{|c|c|c|c|c|c|c|c|}
\hline & Sexo/SIMCE & $\mathrm{N}$ & Media & $\begin{array}{c}\text { Desviación } \\
\text { típ. }\end{array}$ & $\begin{array}{l}\text { Error típ. de } \\
\text { la media }\end{array}$ & $\begin{array}{c}\text { \% alumnos } \\
\text { bajo media } \\
\text { esperada }\end{array}$ & $\begin{array}{c}\text { Media } \\
\text { esperada } \\
\text { en el test }\end{array}$ \\
\hline \multirow{4}{*}{ AES } & Mujer alto SIMCE & 139 & 22,81 & 5,224 & ,443 & $66,2 \%$ & \multirow{4}{*}{25} \\
\hline & Hombre alto SIMCE & 97 & 20,94 & 5,465 & ,555 & $75,3 \%$ & \\
\hline & Mujer bajo SIMCE & 74 & 21,81 & 5,246 & ,610 & $71,6 \%$ & \\
\hline & Hombre bajo SIMCE & 87 & 21,06 & 4,568 & ,490 & $79,3 \%$ & \\
\hline \multirow{4}{*}{ AEV } & Mujer alto SIMCE & 139 & 23,60 & 4,922 & ,417 & $58,3 \%$ & \multirow{4}{*}{25} \\
\hline & Hombre alto SIMCE & 97 & 22,22 & 4,852 & ,493 & $71,1 \%$ & \\
\hline & Mujer bajo SIMCE & 74 & 24,03 & 5,123 & ,596 & $56,8 \%$ & \\
\hline & Hombre bajo SIMCE & 87 & 23,28 & 4,419 & ,474 & $57,5 \%$ & \\
\hline \multirow{4}{*}{ PPE } & Mujer alto SIMCE & 139 & 27,91 & 4,580 & ,388 & $61,2 \%$ & \multirow{4}{*}{30} \\
\hline & Hombre alto SIMCE & 97 & 28,70 & 4,984 & ,506 & $53,6 \%$ & \\
\hline & Mujer bajo SIMCE & 74 & 27,78 & 5,303 & ,616 & $62,2 \%$ & \\
\hline & Hombre bajo SIMCE & 87 & 27,68 & 4,684 &, 502 & $62,1 \%$ & \\
\hline
\end{tabular}

En la Tabla 4, se puede apreciar el puntaje promedio obtenido en el IEEA de acuerdo al sexo del total de la muestra de estudiantes (1 = mujer; 2 = hombre). También, se evidencia que hombres y mujeres tienen puntajes sobre la media esperada en las escalas de concentración. Las mujeres presentan un puntaje sobre la media esperada en la escala de administración del tiempo. En general, hombres y mujeres demuestran un puntaje bajo la media esperada en actitud e interés, motivación, procesamiento de la información, selección de ideas principales, ayudas de estudio, autoevaluación, estrategias para la preparación y presentación de exámenes, y ansiedad, siendo los puntajes de esta última menor en las mujeres, lo que indica que ellas se mostrarían más ansiosas ante los estudios. 
Tabla 4

Resultados descriptivos por género respecto de medias.

\begin{tabular}{|c|c|c|c|c|c|c|c|}
\hline & Género & $\mathrm{N}$ & Media & Desviación típ. & $\begin{array}{l}\text { Error típ. de la } \\
\text { media }\end{array}$ & $\begin{array}{l}\% \text { alumnos } \\
\text { bajo media } \\
\text { esperada }\end{array}$ & $\begin{array}{c}\text { Media } \\
\text { esperada en } \\
\text { el test }\end{array}$ \\
\hline \multirow{2}{*}{ ACT } & 1 & 213 & 27,79 & 5,566 & ,381 & $77,0 \%$ & \multirow{2}{*}{32} \\
\hline & 2 & 184 & 27,43 & 5,384 & ,397 & $76,6 \%$ & \\
\hline \multirow{2}{*}{ MOT } & 1 & 213 & 26,75 & 4,853 & ,333 & $76,5 \%$ & \multirow{2}{*}{31} \\
\hline & 2 & 184 & 25,71 & 4,900 & 361 & $86,4 \%$ & \\
\hline \multirow{2}{*}{ ATI } & 1 & 213 & 23,80 & 5,656 & ,388 & $36,2 \%$ & \multirow{2}{*}{23} \\
\hline & 2 & 184 & 22,98 & 4,661 & ,344 & $47,8 \%$ & \\
\hline \multirow{2}{*}{ ANS } & 1 & 213 & 22,09 & 5,147 & ,353 & $73,2 \%$ & \multirow{2}{*}{26} \\
\hline & 2 & 184 & 23,93 & 5,013 & ,370 & $63,6 \%$ & \\
\hline \multirow{2}{*}{$\mathrm{CON}$} & 1 & 213 & 25,25 & 7,411 &, 508 & $47,4 \%$ & \multirow{2}{*}{25} \\
\hline & 2 & 184 & 28,16 & 9,282 & ,684 & $35,9 \%$ & \\
\hline \multirow{2}{*}{ PIN } & 1 & 213 & 26,32 & 5,381 & 369 & $50,7 \%$ & \multirow{2}{*}{27} \\
\hline & 2 & 184 & 26,68 & 5,055 & 373 & $56,0 \%$ & \\
\hline \multirow{2}{*}{ SIP } & 1 & 213 & 17,48 & 3,021 & 207 & $50,7 \%$ & \multirow{2}{*}{18} \\
\hline & 2 & 184 & 17,47 & 3,327 & 245 & $48,9 \%$ & \\
\hline \multirow{2}{*}{ AES } & 1 & 213 & 22,46 & 5,241 & ,359 & $68,1 \%$ & \multirow{2}{*}{25} \\
\hline & 2 & 184 & 20,99 & 5,048 & ,372 & $77,2 \%$ & \\
\hline \multirow{2}{*}{ AEV } & 1 & 213 & 23,75 & 4,985 & ,342 & $57,7 \%$ & \multirow{2}{*}{25} \\
\hline & 2 & 184 & 22,72 & 4,670 & 344 & $64,7 \%$ & \\
\hline \multirow{2}{*}{ PPE } & 1 & 213 & 27,87 & 4,831 & ,331 & $61,5 \%$ & \multirow{2}{*}{30} \\
\hline & 2 & 184 & 28,22 & 4,858 & ,358 & $57,6 \%$ & \\
\hline
\end{tabular}

1 = mujer; 2 = hombre

Al revisar la relación entre las escalas medidas por el IEEA y el rendimiento académico en los liceos con alto puntaje SIMCE, se puede observar que a un nivel de $p<0,01$ de significancia estadística, la variable de rendimiento académico de liceos con alto puntaje SIMCE correlaciona positivamente con motivación $(r=$ $0,304)$, administración del tiempo $(r=0,182)$, selección de ideas principales $(r=$ $0,184)$; preparación y presentación de exámenes $(r=0,177)$. Con un nivel de significancia estadística de $p<0.05$ correlacionaron positivamente rendimiento académico con actitud $(r=0,134)$.

Con respecto al análisis dentro de los liceos con bajo SIMCE, se puede apreciar con un $95 \%$ de confianza que no existen correlaciones estadísticamente 
significativas entre las escalas medidas por el IEEA y el rendimiento académico de los estudiantes.

Al comparar las estrategias de aprendizaje según liceos, se evidencia que el liceo 1 y el 2 (ambos con bajo SIMCE) presentan diferencias significativas ( $p<$ $0,05)$ respecto de las medias de las escalas evaluadas por el IEEA en actitud e interés, pues el establecimiento 1 posee un puntaje por sobre la media esperada en la escala ( $t=6,8 ; p=0,000$; diferencia de medias $=5,72$ ), esto indica que los alumnos pertenecientes al liceo 1, que tienen bajo puntaje SIMCE, utilizan con mayor frecuencia la estrategia de actitud e interés en los estudios.

Por otro lado, se observa que existe una diferencia significativa con respecto a las medias de los liceos 1 (con bajo puntaje SIMCE) y 3 (alto puntaje SIMCE) en la escala de actitud e interés $(t=8,704 ; p=0,000$; diferencia de medias $=$ $8,06)$, administración del tiempo $(t=3,9 ; p=0,000$; diferencia de medias $=3,74)$ y concentración ( $t=3,761 ; p=0,000$; diferencia de medias $=4,72$ ); el liceo 1 presenta un puntaje por sobre la media esperada en las escala de actitud e interés, administración del tiempo y concentración, lo que indica que los alumnos poseen una mayor administración del tiempo en la realización de sus actividades académicas, en conjunto a una mayor concentración y actitud hacia los estudios.

Con respecto a la comparación entre el liceo 1 y el 4, es notorio que existe una diferencia significativa respecto de las medias de los liceos 1 (bajo puntaje SIMCE) y 4 (alto puntaje SIMCE) en la escala de actitud e interés ( $t=6,013 ; p$ $=0,000$; diferencia de medias $=4,85$ ). El liceo 1 tiene un puntaje por sobre la media esperada en esa escala, lo que indica que los alumnos utilizan con mayor frecuencia la estrategia de actitud e interés en los estudios.

Al comparar el liceo 2 (bajo puntaje SIMCE) con el 3 (alto puntaje SIMCE), existe una diferencia significativa entre las medias en la escala de actitud e interés $(t=3,424 ; p=0,001$; diferencia de medias $=2,33)$, administración del tiempo $(t=$ 3,$996 ; p=0,000$; diferencia de medias $=2,53$ ) y concentración ( $t=5,04 ; p=0,000$; diferencia de medias $=5,65)$. A pesar de que el liceo 2 posee mejores puntajes que el liceo 3 , ambos tienen un puntaje bajo la media esperada en cuanto a actitud e interés, lo que quiere decir que en ambos liceos no existe un uso frecuente de dicha escala. En la escala de administración del tiempo y concentración, el liceo 2 presenta puntajes dentro y sobre la media esperada, lo que indica que los alumnos utilizan con mayor frecuencia las escalas mencionadas. 
Al mismo tiempo, queda en evidencia que existe una diferencia significativa respecto de las medias de los liceos 2 (bajo puntaje SIMCE) y 4 (alto puntaje SIMCE) en la escala de concentración ( $t=3,005 ; p=0,003$; diferencia de medias = 3,337 ), siendo el liceo 2 quien la utiliza con mayor frecuencia; sin embargo, ambos centros educativos demuestran un puntaje dentro y sobre la media esperada en esa escala, lo que quiere decir que en ambos liceos se mantiene una concentración adecuada ante los estudios.

Finalmente, se aprecia que existe una diferencia significativa con respecto a las medias de los liceos 3 y 4 (ambos con alto puntaje SIMCE) en la escala de actitud e interés ( $t=-4,926 ; p=0,000$; diferencia de medias $=-3,21$ ) y administración del tiempo $(t=-3,943 ; p=0,000$; diferencia de medias $=-2,37$ ). El liceo 4 posee un puntaje dentro y sobre la media esperada en dichas escalas, lo que quiere decir que los alumnos poseen una mayor administración del tiempo en la realización de sus actividades académicas, en conjunto con una mayor actitud e interés hacia los estudios.

Al comparar entre estrategias de aprendizaje y SIMCE, existe una diferencia significativa respecto de las medias de los puntajes alto y bajo SIMCE en las escalas de actitud e interés $(t=-4,318 ; p=0,000$; diferencia de medias $=-2,37$ ), administración del tiempo $(t=-3,027 ; p=0,003$; diferencia de medias $=-1,60)$ $y$ concentración ( $t=-4,872 ; p=0,000$; diferencias de medias $=-4,09)$. De esta forma, alto y bajo puntaje SIMCE tienen puntajes bajo la media esperada en la escala de actitud e interés, es decir, los alumnos no poseen actitud e interés hacia los estudios. Respecto a las escalas de administración del tiempo y concentración, los alumnos pertenecientes a liceos con bajo puntajes SIMCE tienen un uso más frecuente en la escala.

Al comparar los resultados de las estrategias de aprendizaje según el sexo de los estudiantes de los liceos con alto puntaje SIMCE, se puede apreciar que existe una diferencia significativa en las medias de las mujeres y hombres en la escala de ansiedad $(t=-3,550 ; p=0,000$; diferencia de medias $=-2,41)$. Los hombres tienden a controlar más la ansiedad que las mujeres, a pesar de que tanto mujeres y hombres pertenecientes a liceos con alto puntaje SIMCE demuestran puntajes bajo la media esperada en la escala, es decir, ambos mantienen un débil manejo de la ansiedad.

Por otro lado, en las mujeres y los hombres pertenecientes a liceos con alto y bajo puntaje SIMCE, se puede observar que existe una diferencia significativa 
en las escalas de ansiedad ( $t=-3,550 ; p=0,000$; diferencia de medias $=-2,41$ ) y concentración ( $t=-3,150 ; p=0,002$; diferencia de medias $=-4,88$ ). Los hombres pertenecientes a liceos con alto SIMCE tienen puntajes mayores que las mujeres pertenecientes a liceos con alto SIMCE, a pesar de que ambos se encuentran bajo la media esperada para la escala de ansiedad. Esto indica que los hombres mantienen un mayor control de la ansiedad.

En la escala de concentración, los hombres pertenecientes a liceos con bajo SIMCE presentan puntajes mayores que las mujeres adscritas a liceos con bajo SIMCE, a pesar de que ambos se encuentran sobre la media esperada en la escala. Esto indica que los hombres presentan una mayor concentración y atención en la realización de actividades académicas.

Finalmente, se puede apreciar que existe una diferencia significativa en las medias por sexo en las escalas de ansiedad $(t=-3,595 ; p=0,000$; diferencia de medias $=-1,84)$, concentración $(t=-3,476 ; p=0,001$; diferencia de medias $=-2,91)$ y ayudas de estudio $(t=2,826 ; p=0,005$; diferencia de medias $=1,47)$. Los hombres presentan puntajes mayores que las mujeres en la escala de concentración, a pesar de que ambos géneros se encuentran sobre la media esperada, lo que indica que los varones poseen una mayor concentración y atención en la realización de actividades académicas. De la misma forma, los hombres tienen puntajes mayores que las mujeres en la escala de ansiedad, a pesar de que ambos se encuentren bajo la media esperada, esto refleja que los estudiantes varones de este grupo mantienen un mayor manejo de la ansiedad que las mujeres. Por otro lado, en la escala de ayudas de estudio, las mujeres presentan puntajes mayores que los hombres, a pesar de que ambos se encuentren bajo la media esperada, es decir, las mujeres demuestran mayor uso de ayudas y técnicas de estudio.

\section{DISCUSIÓN}

Nuestros objetivos e hipótesis generales se cumplieron: Describir la relación que existe entre las estrategias de aprendizaje y rendimiento académico de estudiantes de cuarto año de enseñanza media, adscritos a liceos municipalizados con alto y bajo puntaje SIMCE, pertenecientes a la comuna de Talca. Al respecto, se obtuvieron datos significativos sobre las diferencias entre los alumnos pertenecientes a liceos con alto y bajo puntaje SIMCE. Por otro lado, se determinó que esos resultados se encuentran influenciados por ciertas características como el género, rendimiento académico y puntaje SIMCE. 
De acuerdo con los resultados descriptivos, los alumnos pertenecientes a los liceos 1, 2 y 4 municipalizados de la comuna de Talca muestran un nivel adecuado en el uso de las estrategias de aprendizaje con respecto a la administración del tiempo y a la concentración, sobresaliendo solo el liceo 1 en la escala de actitud e interés hacia los estudios, pues tuvo un puntaje más elevado que el resto en dicha escala. Por otro lado, queda en evidencia un nivel poco adecuado en el uso de las estrategias de aprendizaje en las escalas de motivación, procesamiento de la información, selección de ideas principales, ayudas de estudio, autoevaluación, estrategias para la preparación y presentación de exámenes y, ansiedad, siendo esta última uno de los factores que podría estar influyendo en el uso de las estrategias de aprendizaje. Esto puede estar asociado con los resultados reflejados por el estudio realizado por Muñoz (2005), quien sostiene que los niveles de ansiedad podrían interferir en el proceso de enseñanza aprendizaje de los estudiantes.

Sobre los liceos con alto y bajo puntaje SIMCE, se puede afirmar que los alumnos de los liceos con bajo puntaje SIMCE reflejaron, de acuerdo al análisis descriptivo, datos inesperados, pues presentaron un manejo adecuado de las estrategias de aprendizaje en lo que respecta a administración del tiempo y concentración, a diferencia de los alumnos pertenecientes a los liceos de alto puntaje SIMCE, quienes no sobresalieron en ninguna de las escalas evaluadas por el test.

Con respecto al rendimiento académico, se puede afirmar que no existe relación entre el rendimiento de los liceos con bajo puntaje SIMCE y las escalas evaluadas por el IEEA, contrariamente a lo que ocurre con el rendimiento académico de los estudiantes pertenecientes a liceos con alto puntaje SIMCE, quienes presentan una relación significativa con las escalas de actitud, motivación, administración del tiempo, selección de ideas y preparación y presentación de exámenes. De esta manera, se deja entrever que existe correlación entre estrategias de aprendizaje y buen rendimiento académico en los estudiantes de liceos con alto puntaje SIMCE; estos resultados coinciden con lo planteado en el estudio realizado por Pizano (2004), en el que se menciona que existe una relación estadísticamente significativa entre estrategias de aprendizaje y rendimiento académico, dicho de otra manera, el uso de estrategias de aprendizaje le entrega al alumno la potencialidad de controlar y regular sus procesos mentales de aprendizaje, lo que permitiría mejorar su rendimiento académico y su capacidad de aprender autónomamente (Andrade, 2001). 
De acuerdo con lo anterior, se cumple una de las hipótesis de nuestro estudio: A mayor rendimiento académico, mejor puntaje en al menos una de las subescalas del Inventario de Estrategias y Aprendizaje (IEEA) de los alumnos de cuarto año de enseñanza media de liceos con alto puntaje SIMCE. Sin embargo, la hipótesis: A mayor rendimiento académico, mejor puntaje en al menos una de las subescalas del Inventario de Estrategias y Aprendizaje (IEEA) de los alumnos de cuarto año de enseñanza media, de liceos con bajo puntaje SIMCE no se observa. Esto indica que los estudiantes de liceos con bajo SIMCE, si bien manifiestan mejores puntajes en el IEEA, este uso de estrategias de aprendizaje no presenta relación con el rendimiento académico, al contrario de los estudiantes de liceos con alto SIMCE, quienes realmente demuestran una asociación entre estrategias de aprendizaje y rendimiento académico.

A pesar de las diferencias mencionadas, los alumnos de los distintos establecimientos (alto y bajo puntaje SIMCE) presentan un uso poco frecuente de las estrategias de aprendizaje evaluadas por el IEEA. De acuerdo a esto, se refleja la relación que existe entre las estrategias de aprendizaje y rendimiento académico de alumnos de cuarto año de enseñanza media de liceos con alto puntaje SIMCE en la comuna de Talca.

De igual manera, las hipótesis específicas se confirmaron en su totalidad, dejando de manifiesto que sí existe una relación entre el rendimiento de los liceos con alto puntaje SIMCE y el uso frecuente de las estrategias de aprendizaje, al contrario de los liceos con bajo puntaje SIMCE, quienes no presentaron relación entre dichas variables y rendimiento académico, es decir, si bien reportan mejores estrategias que los estudiantes de liceos con alto puntaje SIMCE, no se correlaciona con el rendimiento académico. Conjuntamente con esto, queda establecida la diferencia significativa existente entre los estudiantes de cuarto medio de liceos municipales con alto y bajo SIMCE, con respecto a los puntajes de las estrategias de aprendizaje, pues los liceos con bajo puntaje SIMCE obtuvieron mayor puntaje en las escalas de administración del tiempo y concentración, mas no así los liceos con alto puntaje SIMCE, quienes no obtuvieron ningún puntaje sobre la media esperada en cuanto a las escalas evaluadas por el IEEA.

Al revisar las estrategias de aprendizaje utilizadas entre los hombres y mujeres con alto y bajo SIMCE, se destaca que tanto los estudiantes hombres con alto puntaje SIMCE y las mujeres con bajo puntaje SIMCE utilizan con mayor frecuencia las estrategias de administración del tiempo, concentración y procesamiento de la información. Lo anterior puede deberse, en el caso de los hombres pertenecientes 
a los liceos con alto SIMCE, a que presentan mayores exigencias, tanto por parte del establecimiento como a nivel personal, lo que los lleva a desarrollar y utilizar con mayor frecuencia las mencionadas estrategias.

En el caso de las mujeres adscritas a los liceos con bajo SIMCE, se podría hipotetizar que la utilización frecuente de dichas estrategias forman parte de la exigencia personal, más que de las exigencias académicas requeridas por el establecimiento; sin dejar de mencionar la relevancia e influencia que puede alcanzar la formación entregada por cada establecimiento a su alumnado.

La diferencia de los resultados obtenidos se puede deber a lo mencionado por Pozo, Monereo \& Castelló (2001), Badenier (2002) y Muñoz (2005), quienes señalan que las estrategias de aprendizaje se relacionan con el contexto en el cual se sitúa el aprendizaje, sin dejar de mencionar la importancia de la autocrítica que se realice el sujeto, ya que de esta depende el grado de autoevaluación que se haga y, por ende, de la exigencia personal que se mantenga, es decir, se alude al rol protagónico del aprendiz, coincidiendo con Pizano (2004). Los autores plantean que el alumno es un agente activo, que construye sus propios conocimientos inteligentemente, y es responsable de la calidad y profundidad del aprendizaje que realice. Conjuntamente con ello, es importante mencionar la etapa de desarrollo en la cual se encuentran estos jóvenes, pues es una etapa de cambios y toma de decisiones, lo que podría generar una inestabilidad personal, la cual influye en el interés y dedicación en los estudios, llevando en mucho de los casos a un falta de conocimiento de sí y por ende de las propias capacidades y habilidades individuales (Surís, 2001).

Al considerar nuestra tercera hipótesis: Se presenta diferencia significativa entre los estudiantes de cuarto medio de liceos municipales con alto y bajo SIMCE, con respecto a los puntajes de las estrategias de aprendizaje. Se comprueba que los estudiantes pertenecientes a liceos con alto puntaje SIMCE utilizan con mayor frecuencia las estrategias de administración del tiempo y concentración en la realización de actividades académicas, a diferencia de los estudiantes pertenecientes a liceos con bajo puntaje SIMCE, quienes también emplean las estrategias de administración del tiempo y concentración en la realización de actividades académicas, pero utilizan con mayor frecuencia la escala correspondiente a la estrategia de actitud e interés hacia los estudios. La razón de esto se puede deber a que los alumnos de liceos con bajo SIMCE presentan asignaturas que generan interés en los alumnos, pues llevan consigo una inmediata aplicación práctica, a diferencia de los alumnos de liceos con alto 
puntaje SIMCE, quienes cuentan con asignaturas basadas en lo teórico, lo cual genera un falta de interés por parte de los alumnos y su actitud hacia ellas se torna displicente.

Con respecto a la relación entre las estrategias de aprendizaje y género, se puede afirmar que los hombres presentan mayor concentración ante los estudios que las mujeres, quienes, a diferencia de los hombres, tienen mayor ayudas de estudio, a pesar de poseer un menor control de la ansiedad, lo que puede influir directamente en el uso de las otras estrategias de aprendizaje. Sin embargo, tanto hombres como mujeres evidencian un elevado nivel de ansiedad frente a los estudios, lo cual concuerda con lo planteado por Pintrich y García (citados por Rinaudo, Chiecher y Donolo, 2003) cuando postulan que la ansiedad se correlaciona negativamente con el uso de las estrategias de aprendizaje.

Se puede inferir que, tanto los hombres como mujeres pertenecientes a liceos con bajo puntaje SIMCE utilizan con mayor frecuencia la escala de administración del tiempo y concentración, siendo esta última presentada habitualmente por los hombres pertenecientes a liceos con alto puntaje SIMCE, quienes, a su vez, emplean con mayor frecuencia el procesamiento, adquisición y razonamiento de la información. De este modo, se acepta la hipótesis que indica que: Se presentan diferencias significativas entre las estrategias de aprendizaje y el género de los estudiantes de cuarto año de enseñanza media de liceos municipalizados con alto y bajo puntaje SIMCE.

Inversamente, entre las escalas utilizadas con menor frecuencia o ausentes, se encuentran las escalas de actitud e interés y motivación, las cuales son relacionadas por lo planteado por Rinaudo, Chiecher y Donolo (2003), quienes muestran una vinculación entre la motivación y el interés que genera una determinada actividad académica, considerada como un fin en sí misma y no como un medio para alcanzar otras metas.

Es importante aclarar que, como limitadores del estudio realizado se puede mencionar la selección de la muestra, pues en una primera instancia correspondería a liceos científicos humanistas municipalizados con alto y bajo puntaje SIMCE, pero no constituían una muestra significativa de los liceos de la comuna de Talca. Por ello, se optó por la incorporación de liceos con formación técnico profesional. Sumado a esto, otra limitante estuvo constituida por la poca disponibilidad temporal de los establecimientos educativos para la aplicación del inventario, incluso en los liceos 2, 3 y 4, por encontrarse cercanos al término de semestre, no contaban con 
fechas disponibles debido al período de evaluaciones. Esto atrasó el proceso de recolección de datos. A pesar de lo anterior, es importante recalcar que todos los liceos evaluados mostraron gran interés en el trabajo, ya que les agradó la idea de conocer las estrategias de aprendizaje utilizados por los alumnos pertenecientes a cada liceo.

Además, es importante mencionar que, a pesar de ser una muestra numerosa, la población representa solo a liceos municipalizados de la comuna de Talca, por ende, se desconoce si los resultados arrojados por este estudio son representativos a nivel regional y nacional, razón por la cual se sugiere la realización de investigaciones a nivel nacional con respecto a la relación existente entre estrategias de aprendizaje y rendimiento académico de estudiantes que pertenezcan a liceos particulares-subvencionados y particulares, con alto y bajo puntaje SIMCE. De esa manera, se podrá verificar o contrastar los resultados obtenidos en el presente estudio, para posteriormente fomentar y reforzar aquellas áreas que se encuentren débiles respecto del proceso enseñanza-aprendizaje.

Finalmente, se sugiere investigar la influencia de otras variables en el uso de las estrategias de aprendizaje como: autoconcepto de los estudiantes, instrucciones estratégicas implícitas en los diferentes contenidos disciplinares, programas de entrenamiento, diversidad de muestras y estudios longitudinales en el sistema educativo secundario y universitario. Esta sería una forma de avanzar en la búsqueda de mayor eficacia y eficiencia académica de los estudiantes.

Por otro lado, dentro de las propuestas que se consideran claves para trabajar las estrategias de aprendizaje de los alumnos, es importante destacar la implementación de un programa de intervención, tanto para alumnos como padres y docentes, el cual debe estar basado en el modelo de Weinstein y Mayer (citado en Badenier, 2002). Ese programa propone seis categorías de estrategias de aprendizaje: (1) de repetición, que puede ser utilizada de mejor manera cuando el material a ser aprendido es una lección, un libro, una prosa, etc., mediante la repetición en voz alta, la toma de notas selectivas, el copiado y subrayado de las parte de mayor importancia del material; (2) de elaboración, cuya meta es la integración de la información presentada con el conocimiento previo y la creación de imágenes mentales que permitan la conexión entre dos o más conceptos, a través de la creación de analogías, toma de notas y realización de preguntas y resúmenes; (3) de selección; y (4) de organización, que a la vez, presenta dos mentas, la selección de información y la construcción de relaciones entre ideas. Esta última categoría puede lograrse mediante la organización del material y la 
construcción de esquemas simbólicos y diagramas; (5) de control de la comprensión, que busca que los estudiantes se establezcan metas de aprendizaje para una unidad 0 actividad instruccional, a través de las habilidades de autointerrogación (“¿Qué tengo que hacer?"), autopreguntas ("Cuidadosamente, me detengo y vuelvo a leer el párrafo"), autoreforzamiento ("Sí, estoy entendiendo el párrafo"), y enfrentamiento de los errores ("Voy bien, a pesar de que me cuesta comprender, seguiré leyendo"); y (6) las afectivas, que incluyen el estar alerta, relajado y lejos de factores distractores para enfrentar de mejor manera una prueba, a través de la atención focalizada, el mantenimiento de la concentración, el manejo de las conductas ansiosas, el establecimiento y mantención de la motivación y el manejo efectivo del tiempo. Este entrenamiento cognitivo es conducido a través de las tareas, el ambiente y las personas involucradas en el proceso de enseñanza aprendizaje (alumnos, docentes y padres), quienes debieran adoptar un rol activo en la implementación, desarrollo y mantenimiento del mencionado modelo.

En todo caso, convendría la realización de talleres mediante trabajos individuales e instancias de discusión con respecto a los propios procesos de aprendizaje, abarcando: los hábitos de estudio mediante el establecimiento de horarios, formación de grupos de estudio, formas de procesar mejor la información y el uso adecuado de estrategias de aprendizaje, de acuerdo a aquellas que concuerden con las asignaturas de menor rendimiento, a partir de la consideración del contexto en el que se desarrollan las diversas asignaturas. También, es necesario crear espacios para generar metareflexiones con respecto al cómo se está aprendiendo e instancias de aprendizaje para la autorregulación y responsabilidad acerca del propio aprendizaje. De igual manera, resulta de gran importancia relacionar el aprendizaje del aula con la vida cotidiana y ayudar a que los alumnos enfrenten los exámenes de forma tranquila y segura, además de reforzar y estimular la motivación durante el aprendizaje, ya que es un factor bastante influyente al momento de estudiar.

También es necesario trabajar con los estudiantes la ansiedad, a través de talleres de relajación y respiración, con la finalidad de disminuir los niveles de esta y de ejercer un mejor control sobre ella. Por otro lado, es importante fomentar y adiestrar en los escolares desde el nivel básico el uso las estrategias basadas en la autoevaluación; así como en el grado de: interés, motivación (intrínseca y extrínseca), concentración, adquisición, razonamiento y procesamiento de la información, pues todos esos factores tienen una incidencia positiva en el rendimiento académico de los estudiantes. 


\section{REFERENCIAS BIBLIOGRÁFICAS}

ANDRADE, M. (2001). Teorías del aprendizaje y estrategias cognitivas: Una perspectiva teórica. Programa de Postítulo en subsectores de Aprendizaje. Santiago: Editorial Crear. (pp.120-129).

BADENIER, C. (2002). Confiabilidad y validez del Learning and Study Strategies Inventory (LASSI) en una muestra de estudiantes de la región metropolitana. Tesis presentada a la Escuela de Psicología de la Pontificia Universidad Católica de Chile, como requisito para optar al grado de Magíster en Psicología Educacional.

CABRERA, E., GARCÍA, L., TORBAY, Á. Y RODRÍGUEZ, T. (2007). Estructura factorial y fiabilidad de un cuestionario de estrategias de aprendizaje en universitarios: CEA-U. Revista Anales de Psicología, Vol. 23, nº 1. (pp. 1-6).

MINEDUC (2006). Sistema de Medición de la Calidad Educativa (SIMCE). Extraído el 31 de Octubre del 2007 de http://www.simce.cl/index.php?id=91

MONEREO, C., POZO, J. Y CASTELLÓ, M. (2004). La enseñanza de estrategias de aprendizaje en el contexto escolar. En Coll, C., Palacios, J., y Marchesi, A. (comp.), Desarrollo psicológico y educación: psicología de la educación escolar. Madrid: Editorial Alianza. (pp. 235-258).

MUÑOZ, M. (2005). Estrategias de aprendizaje en estudiantes universitarias. Extraído el 25 de octubre del 2006 de http://www.psicologiacientifica.com/bv/ psicologia-62-1-estrategias-de-aprendizaje-en-estudiantes-universitarias.html

MUÑOZ, M. Y LUCERO, B. (2006). Salud mental y estrategias de aprendizaje en estudiantes de psicología. XIX Encuentro Nacional y V Internacional de Investigadores en Educación, del 14 al 16 de noviembre CPEIP. Santiago de Chile.

PIZANO, G. (2004). Las Estrategias de Aprendizaje y su relación con el rendimiento académico de los alumnos. Revista electrónica de Investigación Educativa. Año $8^{\circ}$, nº 14, pp.27-30.pp.28. Extraído el 14 de noviembre del 2007 desde http:// sisbib.unmsm.edu.pe/bibvirtualdata/Publicaciones/inveducativa/2004n14/a03.pdf

PROCTOR, B., PREVATT, F., ADAMS, K., HURST, A. \& PETSCHER, Y. (2006). Study Skills Perfiles of Normal-Achieving and Academically-Struggling College Students". Revista Project Muse, Vol. 47, ํo 1. 
RINAUDO, M., CHIECHER, A. Y DONOLO, D. (2003). Motivación y uso de estrategias en estudiantes universitarios: Su evaluación a partir del Motivated Strategies Learning Questionnaire. Revista Anales de Psicología, Vol. 19, no 1, pp. 107-119.

Surís, J. (2001). Un adolescente en casa. España: Editorial Bolsillo.

WOOLFOLK, A. (2015). Psicología educativa. México: Editorial Pearson.

ZULIA, C. (2006). Estrategias de aprendizaje cognitivas y metacognitivas activadas por los estudiantes de enfermería en la asignatura de farmacología. Extraído el 05 de octubre del 2006 de http://www.monografías.com/trabajos/estrategiasaprendizaje/estrategias-aprendizaje.shtml?monosearch 